\title{
Efeitos do Ácido L-Glutâmico da Dieta em Pintos de Corte ${ }^{1}$
}

\author{
Fernanda Alvares da Silva², George Henrique Kling de Moraes ${ }^{2}$
}

\begin{abstract}
RESUMO - Com o objetivo de estudar os efeitos de diferentes níveis de ácido L-glutâmico (L-Glu) sobre as concentrações de proteínas colagenosas (PC), proteínas não-colagenosas (PNC), proteínas totais (PT), cálcio $(\mathrm{Ca})$, fósforo $(\mathrm{P})$, magnésio $(\mathrm{Mg})$, manganês $(\mathrm{Mn})$, sódio $(\mathrm{Na})$ e potássio $(\mathrm{K})$ nos fêmures e tíbias, um experimento foi conduzido com pintos de um dia, machos, Hubbard, criados em baterias aquecidas com piso de tela elevado, alimentados com dieta básica purificada contendo todos os L-aminoácidos essenciais, vitaminas e minerais em níveis requeridos e suplementada com 5; 7,5; 10; e 12,5\% de ácido L-glutâmico (L-Glu), por um período de 14 dias. O delineamento experimental foi o inteiramente casualizado, com quatro tratamentos, cinco repetições de cinco aves cada. Os pintos alimentados com 7,5\% de L-Glu apresentaram maior ganho de peso, melhor eficiência alimentar e maiores comprimentos de tíbias e fêmures. Foram necessários $12,5 \%$ de L-Glu para reduzir a um mínimo (19,92\%) a incidência de deformações nas pernas, sem contudo eliminá-las. Não foi observado efeito dos níveis de L-Glu no consumo ( $\mathrm{P}>0,05)$. Não houve efeito significativo dos tratamentos sobre os teores de PC, PT, Ca, P, Mg, Mn, Na e K nas tíbias e fêmures. Os teores de PNC das tíbias e fêmures foram significativamente maiores nos animais alimentados com 12,5\% de L-Glu. Embora algumas diferenças tenham sido observadas na composição mineral dos ossos, os conteúdos se encontravam numa faixa fisiológica normal.
\end{abstract}

Palavras-chave: ácido L-glutâmico, composição química e bioquímica, pintos de corte, problemas de pernas

\section{Effects of Dietary L-Glutamic Acid in Broiler Chicks}

\begin{abstract}
In order to study the effects of L-glutamic acid (L-Glu) levels on contents of collagenous (CP), non collagenous (NCP) total proteins (TP) and minerals in femur and tibia of male broiler chicks, one experiment was conducted with day-old broiler chicks, male Hubbard, reared in electrically heated batteries with raised wire mesh floors and fed purified amino acid diets and water ad libitum for a 14 days period. The basal diet contained all essential L-amino acids, minerals and vitamins at requirement levels and was supplemented with 5,0; 7,5; 10,0; 12,5\% L-Glutamic acid (L-Glu). Each treatment had five replicates of five chicks each. Supplementation of $7.5 \%$ L-Glu was sufficient to improve growth, feed/gain ratio and the higher tibia and femur length. To reduce leg problems to a minimum observed (19.92\%) were necessary higher level of non-specific nitrogen (12,5\% L-Glu). No effects were observed of L-Glu levels upon feed consumption. There was no significant dietary effect in the content of CP, TP and Ca, P, Mg, Mn, Na e K tibia and femur ash. The NCP contents of both the tibia and femur were significant higher in chicks fed 12,5\% L-Glu. Increasing the dietary level of non-specific nitrogen, it was observed a tendency to increase NCP content of tibia and femur. Although some differences were observed in the mineral composition of bones, the mineral contents were in physiological range.
\end{abstract}

Key Words: broiler chicks, L-glutamic acid, chemical and biochemical composition, leg problems

\section{Introdução}

A ciência avícola evoluiu consideravelmente nos últimos tempos, em todos os seus aspectos, transformando a atividade de produção de aves e ovos em uma grande indústria de alimentos (Honma, 1992).

Dados dos últimos 20 anos revelam que o peso corporal de frangos aos 56 dias de idade passou de 1,6 a 3,0 kg. Durante o mesmo período, o crescimento do músculo peitoral maior aumentou numa velocidade mais rápida que a do peso corporal (Lilburn, 1994).

As mudanças no crescimento e desenvolvimento do esqueleto têm contribuído para o aumento da incidência de "fraqueza de pernas". Esses problemas estruturais levam a distorção e deformação de ossos das pernas, sendo mais observados durante a fase posterior de crescimento, provavelmente devido ao ganho de peso corporal e desenvolvimento desproporcional do músculo peitoral. Essa hipótese tem sido proposta por Nestor et al. $(1985 ; 1987)$, citados por Lilburn (1994).

De acordo com Moraes et al. (1984), Ribeiro et al. (1995a, b), Rodrigues \& Moraes (1995) e Rodrigues et al. (1996), as deformações ósseas das pernas podem aparecer em razão de uma deficiência de nitrogênio não-específico. Os teores de cinzas, cál- 
cio, fósforo e magnésio não têm sido alterados de maneira contundente em fêmures e tíbias, mas tem sido observada alteração nos teores de proteínas não-colagenosas em ambos os ossos, que tem permitido sugerir que as deformações possam estar relacionadas com a matriz orgânica dos ossos (Tafuri et al., 1993; Rodrigues et al., 1996). Pouco se sabe sobre a composição química total ou mesmo parcial de ossos das pernas de animais deficientes em nitrogênio nãoespecífico. Além disso, as pequenas variações em teores de macrominerais ósseos têm permitido sugerir que as deformações nas pernas estejam associadas a alterações na matriz orgânica dos ossos.

Assim, foi objeto de estudo testar níveis crescentes de ácido L-glutâmico, como fonte de nitrogênio não-específico, em dietas purificadas, sobre o desempenho, problemas de pernas e a composição bioquímica e química de ossos das pernas de pintos de corte, a fim de determinar um perfil dos teores de proteínas e minerais de animais com ou sem deformações nas pernas.

\section{Material e Métodos}

Um experimento foi conduzido utilizando pintos de um dia, machos, Hubbard, por um período de 14 dias, distribuído em um delineamento inteiramente casualizado, consistindo de quatro tratamentos com cinco repetições de cinco aves cada. Os animais foram criados em baterias aquecidas com piso de tela elevado, recebendo dieta e água à vontade no Setor de Avicultura do Departamento de Zootecnia da Universidade Federal de Viçosa.

As dietas experimentais consistiram em dieta básica purificada (Guimarães, 1988) (Tabela 1), contendo todos os L-aminoácidos essenciais, vitaminas (Roche) e minerais, suplementada com quatro níveis de L-Glu $(5,0 ; 7,5 ; 10,0$; e 12,5\%).

Foram determinados, ao término da fase experimental, ganho de peso, consumo alimentar, eficiência alimentar, comprimentos de tíbias e fêmures e incidência de problemas de pernas. Os problemas de pernas foram avaliados com os animais em caminhada na bateria, por quatro pessoas, e classificados como moderados e severos, de acordo com a intensidade da anomalia. Os dados referentes a esses problemas foram transformados em arc.senv\%. As médias foram comparadas pelo teste de Newman-Keuls a $5 \%$ de significância.

R. Bras. Zootec., v.31, n.1, p.411-416, 2002 (suplemento)
Tabela 1 - Composição da dieta básica purificada Table 1 - Composition of the purified diet

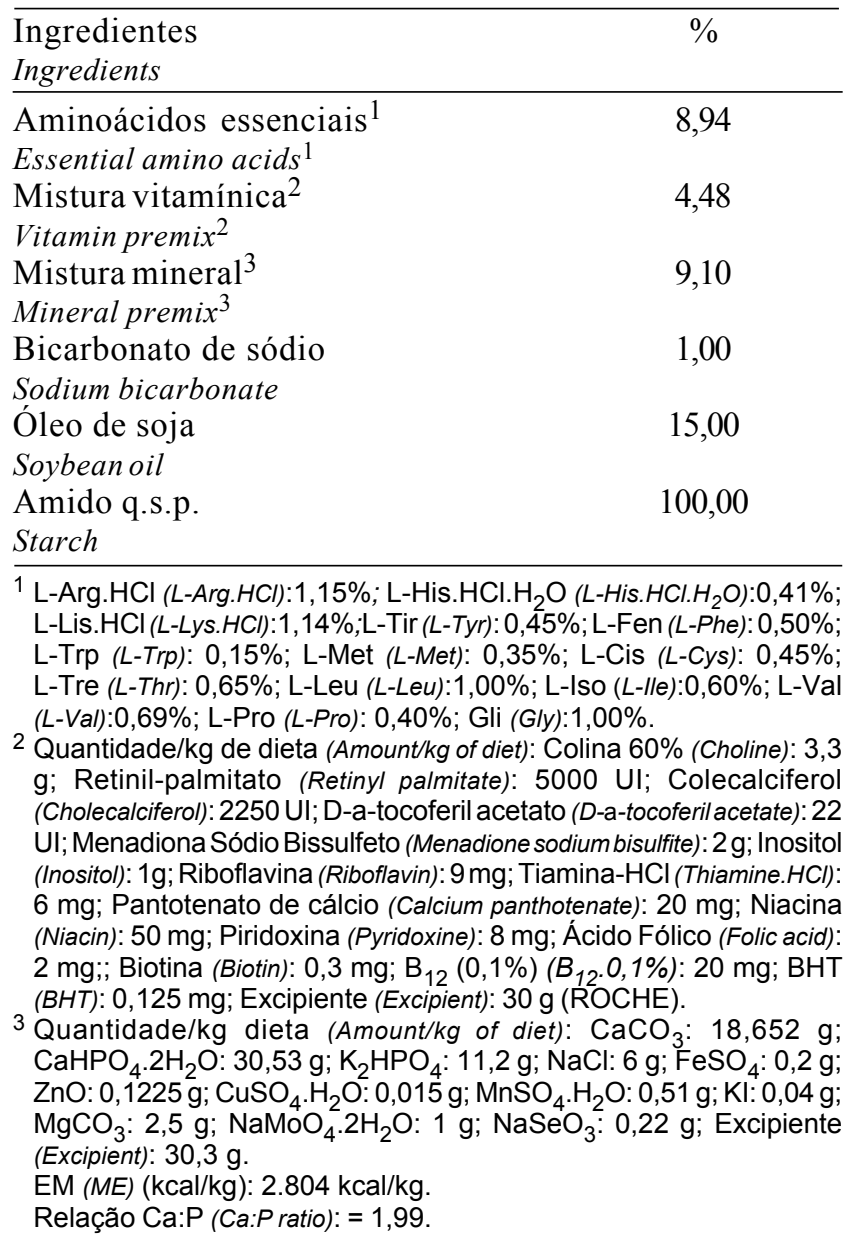

Oito aves de cada tratamento foram sacrificadas por deslocamento cervical. As tíbias e os fêmures de ambas as pernas foram removidos, limpos de todo tecido aderente e cortados longitudinalmente, sendo a medula retirada com jatos de água destilada e deionizada. Os ossos foram, então, desengordurados por 18 horas com éter de petróleo, em aparelho de Soxhlet. Em seguida, foram colocados em sacos plásticos, etiquetados e acondicionados em congelador até o momento das análises químicas e bioquímicas, conduzidas no Laboratório de Bioquímica Animal do Departamento de Bioquímica e Biologia Molecular da Universidade Federal de Viçosa.

As tíbias e os fêmures de uma das pernas foram utilizados para determinação de proteínas nãocolagenosas (PNC), proteínas colagenosas (PC) e proteínas totais $(\mathrm{PT})$.

Cinco ossos de cada tratamento foram pesados e desmineralizados com volumes constantes de 5,0 mL 
de EDTA, sal dissódico, 0,5 moles/L, pH 8,2, de acordo com Hauschka \& Gallop (1977), para extração das PNC. Os extratos de EDTA obtidos foram utilizados na determinação dos teores de PNC, pelo método de Bradford (1976), utilizando a albumina sérica bovina como padrão.

Os ossos foram lavados com água destilada e deionizada, para eliminar o EDTA, e utilizados na determinação dos teores de PC. Este método foi proposto por Berthelot para estimar o teor de nitrogênio, sendo modificado por Pezemek e Nielsen (Guimarães, 1993a). O teor de PC foi obtido multiplicando-se o teor de nitrogênio pelo fator 6,25 . Os teores de PT foram obtidos pela soma dos teores de PNC e PC encontrados anteriormente.

As tíbias e os fêmures da outra perna foram utilizados na determinação dos teores de cálcio $(\mathrm{Ca})$, fósforo $(\mathrm{P})$, magnésio $(\mathrm{Mg})$, manganês $(\mathrm{Mn})$, sódio $(\mathrm{Na})$ e potássio $(\mathrm{K})$ nas cinzas. Os teores de $\mathrm{Ca}, \mathrm{Mg}, \mathrm{Mn}, \mathrm{Na}$ e $\mathrm{K}$ foram determinados na solução mineral, após devidamente diluída, por espectrofotometria de absorção atômica. Os teores de fósforo foram determinados utilizando-se o método descrito por Silva (1990), usando uma solução-padrão de 10 ppm de fósforo.

Os dados foram submetidos à análise de variância, utilizando o programa SAEG (Sistema de Análises Estatísticas e Genéticas) da Universidade Federal de Viçosa - UFV (1997). As médias foram comparadas pelo teste de Newman-Keuls a 5\% de probabilidade.

\section{Resultados e Discussão}

Os dados referentes a ganho de peso, consumo, eficiência alimentar e problemas de pernas observados nos pintos aos 14 dias de idade são apresentados na Tabela 2.

A partir do nível de 7,5\% de L-Glu não foi observada nenhuma diferença estatística $(\mathrm{P}>0,05)$ no desempenho dos animais. Esse nível foi altamente aproveitado pelos animais como fonte de nitrogênio não-específico para obtenção de bom desempenho e crescimento máximo, mas não foi suficiente para reduzir os problemas de pernas. Foi necessário um nível de $12,5 \%$ de L-Glu para reduzir a um mínimo a incidência de anomalias. Resultados similares foram obtidos por Guimarães et al. (1993a, b), Ribeiro et al. (1995a, b) e Moraes et al. (1997), não sendo verificados casos severos de deformidades.

Elkin et al. (1978) constataram que a substituição de farinha de soja por aminoácidos purificados resultou em aumento acentuado nos problemas de pernas. Usando dietas de aminoácidos purificados, Moraes et al. (1984) mostraram que a utilização de $5 \%$ de ácido L-glutâmico como única fonte de nitrogênio nãoespecífico resultou em grande número de problemas de pernas.

Enquanto a adição de $10 \%$ de L-Glu foi necessária para otimizar o crescimento, e o nível de $12,5 \%$ foi necessário para o ótimo conteúdo da matriz orgânica de ossos da perna e redução dos problemas de pernas. Não foram observadas diferenças nos teores de cinzas ósseas; apenas o conteúdo da matriz orgânica foi influenciado.

Os comprimentos médios de tíbias e fêmures encontram-se na Tabela 3 . Os pintos alimentados com dietas suplementadas com 5\% de L-Glu apresentaram comprimentos de tíbias e fêmures significativamente menores $(\mathrm{P}<0,05)$ do que aqueles alimentados com dieta com os demais níveis de L-Glu. O nível de 7,5\% de L-Glu foi suficiente para o bom crescimento dos ossos da perna durante o período experimental.

Lilburn (1994) observou que o comprimento e a largura de tíbias de frangos são consideravelmente menores quando comparados com os de patos e perus de mesmas idades, sugerindo que os frangos devem

Tabela 2 - Valores médios de ganho de peso, consumo, conversão alimentar e problemas de pernas de pintos de corte, machos, aos 14 dias de idade

Table 2 - Weight gain, feed intake, feed/gain ratio and leg weakness of male broiler chicks at 14 days of age

\begin{tabular}{lcccc}
\hline $\begin{array}{l}\text { L-Glu (\%) } \\
\text { L-Glu (\%) }\end{array}$ & $\begin{array}{c}\text { Ganho de peso } \\
\text { Weight gain }\end{array}$ & $\begin{array}{c}\text { Consumo } \\
\text { Feedintake }\end{array}$ & $\begin{array}{c}\text { Conversão alimentar } \\
\text { Feed/gain ratio }\end{array}$ & $\begin{array}{c}\text { Problemas de pernas* }^{*}(\%) \\
\text { Leg weakness }\end{array}$ \\
\hline 5,0 & $126,83^{\mathrm{b}}$ & $261,10^{\mathrm{a}}$ & $2,07^{\mathrm{a}}$ & $76,71^{\mathrm{a}}$ \\
7,5 & $183,39^{\mathrm{a}}$ & $299,01^{\mathrm{a}}$ & $1,62^{\mathrm{b}}$ & $78,75^{\mathrm{a}}$ \\
10,0 & $187,24^{\mathrm{a}}$ & $297,47^{\mathrm{a}}$ & $1,58^{\mathrm{b}}$ & $60,85^{\mathrm{a}}$ \\
12,5 & $204,09^{\mathrm{a}}$ & $288,15^{\mathrm{a}}$ & $1,41^{\mathrm{b}}$ & $19,92^{\mathrm{b}}$ \\
\hline
\end{tabular}

* Médias, na coluna, seguidas de letras minúsculas iguais não diferem $(P>0,05)$ pelo teste de Newman-Keuls.

* Means, in column, with the same small letters, do not differ $(P>.05)$ by Newman-Keuls test. 
Tabela 3 - Comprimentos médios de tíbias ${ }^{*}$ e fêmures ${ }^{*}$ de pintos de corte

Table 3 - Tibia and femur length of male broiler chicks at 14 days of age

\begin{tabular}{ccc}
\hline L-Glu $(\%)$ & Tíbias $(\mathrm{mm})$ & Fêmures $(\mathrm{mm})$ \\
L-Glu $(\%)$ & Tibia $(\mathrm{mm})$ & Femur $(\mathrm{mm})$ \\
\hline 5,0 & $48,25^{\mathrm{b}}$ & $34,18^{\mathrm{b}}$ \\
7,5 & $54,31^{\mathrm{a}}$ & $39,87^{\mathrm{a}}$ \\
10,0 & $55,87^{\mathrm{a}}$ & $40,43^{\mathrm{a}}$ \\
12,5 & $53,25^{\mathrm{a}}$ & $38,43^{\mathrm{a}}$ \\
\hline
\end{tabular}

${ }^{*}$ Médias, na coluna, seguidas de letras iguais não diferem $(P>0,05)$ pelo teste de Newman-Keuls.

*Means, in column, with the same small letters, do not differ ( $P>$.05), by NewmanKeuls test.

ter um grande potencial para problemas biomecânicos, que resultariam em distorção do osso.

Os teores de PNC, PC e PT de tíbias e fêmures são apresentados nas Tabelas 4 e 5.

Quando se elevou o nível de L-Glu das dietas acima de $10 \%$ houve aumento nos teores de PNC de fêmures e tíbias. Os resultados demonstraram que o aumento da porcentagem de nitrogênio não-específico da dieta melhorou o desempenho, reduzindo os problemas de pernas com tíbias e fêmures apresentando teores crescentes de proteínas nãocolagenosas (PNC).

Os resultados demonstraram que o aumento da porcentagem de nitrogênio não-específico eleva os teores de PNC ósseas. Por ser o aminoácido g-carboxiglutâmico(Gla) componente da PNC e oriundo da g-carboxilação do L-Glu, esperava-se que um aumento no nível de L-Glu na dieta elevasse o teor de PNC ósseo.

Os teores de PC e PT em fêmures e tíbias dos

Tabela 4 - Teores médios de proteínas não-colagenosas (PNC), colagenosas (PC) e totais (PT) de tíbias de pintos de corte, machos, aos 14 dias de idade

Table 4 - Non collagenous (NCP), collagenous (CP) and total (TP) proteins in the tibia of male broiler chicks, at 14 days of age

\begin{tabular}{cccc}
\hline L-Glu (\%) & $\mathrm{PNC}^{*}$ & $\mathrm{PC}^{*}$ & $\mathrm{PT}^{*}$ \\
L-Glu (\%) & $N C P^{*}$ & $C P^{*}$ & $T P^{*}$ \\
\hline 5,0 & $1,26^{\mathrm{b}}$ & $28,19^{\mathrm{a}}$ & $29,45^{\mathrm{a}}$ \\
7,5 & $1,42^{\mathrm{b}}$ & $25,37^{\mathrm{a}}$ & $26,79^{\mathrm{a}}$ \\
10,0 & $1,37^{\mathrm{b}}$ & $26,60^{\mathrm{a}}$ & $27,97^{\mathrm{a}}$ \\
12,5 & $1,88^{\mathrm{a}}$ & $24,14^{\mathrm{a}}$ & $26,02^{\mathrm{a}}$ \\
\hline
\end{tabular}

${ }^{*}$ Médias, nas colunas, seguidas de letras minúsculas iguais não diferem $(P>0,05)$ pelo teste de Newman-Keuls.

* Means, in column, with the same small letters, do not differ $(P>.05)$ by Newmankeuls test.

R. Bras. Zootec., v.31, n.1, p.411-416, 2002 (suplemento) pintos submetidos às diferentes dietas experimentais não diferiram entre si $(\mathrm{P}>0,05)$. Resultados similares foram obtidos por Guimarães et al. (1993a, b), Ribeiro et al. (1995a) e Rodrigues et al. (1996).

Os teores médios de cálcio, fósforo, magnésio, manganês, sódio e potássio de tíbias e fêmures constam das Tabelas 6 e 7.

Não foi observada nenhuma relação dos teores de $\mathrm{Ca}, \mathrm{P}, \mathrm{Na}, \mathrm{K}, \mathrm{Mg}$, Mn e relação $\mathrm{Ca}: \mathrm{P}$ nas cinzas com o desempenho e com a incidência de anomalias de pernas nos animais alimentados com os diferentes níveis de L-Glu. Essas dietas já apresentavam os níveis de minerais adequados, de acordo com os níveis recomendados. Esses dados sugerem que a incidência de anomalias esteja diretamente relacionada com a fração orgânica do osso, uma vez que a fração inorgânica não foi afetada.

Os teores de $\mathrm{Na}$ e $\mathrm{K}$ das cinzas de tíbias foram influenciados pelos níveis de L-Glu da dieta $(\mathrm{P}<0,05)$. Foi observado aumento no teor de Na com a elevação do nível de L-Glu da dieta, e o teor de $\mathrm{K}$ foi inferior $(\mathrm{P}<0,05)$ nas tíbias de pintos submetidos a 5,0\% de LGlu. Embora as dietas contenham os níveis recomendados destes elementos, os dados obtidos sugerem que houve efeito benéfico do nível de L-Glu da dieta na retenção de $\mathrm{Na}$ e $\mathrm{K}$ nas tíbias. Todavia, níveis iguais ou superiores a 7,5\% de L-Glu da dieta resultaram em teores de $\mathrm{Na}$ e $\mathrm{K}$ similares $(\mathrm{P}>0,05)$.

Não foi observado efeito dos níveis de L-Glu sobre os teores de $\mathrm{Ca}, \mathrm{P}, \mathrm{Mg}, \mathrm{Mn}, \mathrm{Na}$ e K nos fêmures. As diferenças estatísticas observadas para $\mathrm{Na}$ e $\mathrm{K}$ das tíbias e $\mathrm{P}$ dos fêmures não mostraram relação com a incidência de problemas de pernas relatada.

Os aumentos dos teores de PNC não afetaram a

Tabela 5 - Teores médios de proteínas não-colagenosas (PNC), colagenosas (PC) e totais (PT) de fêmures de pintos de corte, aos 14 dias de idade

Table 5 - Non-collagenous (NCP), collagenous (CP), and total (TP) proteins in the femur of male broiler chicks, at 14 days of age

\begin{tabular}{cccc}
\hline L-Glu(\%) & $\mathrm{PNC}^{*}$ & $\mathrm{PC}^{*}$ & $\mathrm{PT}^{*}$ \\
L-Glu (\%) & $N C P^{*}$ & $C P^{*}$ & $T P^{*}$ \\
\hline 5,0 & $0,95^{\mathrm{a}}$ & $25,38^{\mathrm{a}}$ & $26,33^{\mathrm{a}}$ \\
7,5 & $1,11^{\mathrm{ab}}$ & $27,78^{\mathrm{a}}$ & $28,89^{\mathrm{a}}$ \\
10,0 & $1,08^{\mathrm{ab}}$ & $26,02^{\mathrm{a}}$ & $27,10^{\mathrm{a}}$ \\
12,5 & $1,34^{\mathrm{a}}$ & $26,33^{\mathrm{a}}$ & $27,67^{\mathrm{a}}$ \\
\hline
\end{tabular}

* Médias, nas colunas, seguidas de letras minúsculas não diferem $(P>0,05)$ pelo teste de Newman-Keuls.

*Means, in each column, with the same small letters, do not differ (P>.05) by Newman-Keuls test. 
Tabela 6 - Teores médios de cálcio $(\mathrm{Ca})$, fósforo $(\mathrm{P})$, magnésio $(\mathrm{Mg})$, manganês $(\mathrm{Mn})$, sódio $(\mathrm{Na})$ e potássio $(\mathrm{K})$ das cinzas das tíbias de pintos de corte

Table 6 - Calcium (Ca), phosphorus ( $P$ ), magnesium (Mg), manganese (Mn), sodium ( $\mathrm{Na}$ ) and potassium (K) in the tibia of broiler chicks

\begin{tabular}{lcccccc}
\hline L-Glu $(\%)$ & $\mathrm{Ca}^{*}(\%)$ & $\mathrm{P}^{*}(\%)$ & $\mathrm{Mg}^{*}(\%)$ & $\mathrm{Mn}^{*}(\%)$ & $\mathrm{Na}^{*}(\%)$ & $\mathrm{K}^{*}(\%)$ \\
$L-G l u(\%)$ & $C^{*}(\%)$ & $P^{*}(\%)$ & $M^{*}(\%)$ & $M^{*}(\%)$ & $N a^{*}(\%)$ & $K^{*}(\%)$ \\
\hline 5,0 & $30,13^{\mathrm{a}}$ & $14,59^{\mathrm{a}}$ & $0,55^{\mathrm{a}}$ & $0,0072^{\mathrm{a}}$ & $0,68^{\mathrm{b}}$ & $0,60^{\mathrm{b}}$ \\
7,5 & $31,66^{\mathrm{a}}$ & $15,48^{\mathrm{a}}$ & $0,57^{\mathrm{a}}$ & $0,0064^{\mathrm{a}}$ & $0,96^{\mathrm{ab}}$ & $0,81^{\mathrm{a}}$ \\
10,0 & $32,24^{\mathrm{a}}$ & $14,78^{\mathrm{a}}$ & $0,60^{\mathrm{a}}$ & $0,0090^{\mathrm{a}}$ & $1,01^{\mathrm{ab}}$ & $0,82^{\mathrm{a}}$ \\
12,5 & $30,43^{\mathrm{a}}$ & $13,98^{\mathrm{a}}$ & $0,60^{\mathrm{a}}$ & $0,0073^{\mathrm{a}}$ & $1,09^{\mathrm{a}}$ & $0,89^{\mathrm{a}}$ \\
\hline
\end{tabular}

* Médias, nas colunas, seguidas de letras minúsculas não diferem $(P>0,05)$ pelo teste de Newman-Keuls.

* Means, in each column, with the same small letters, do not differ $(P>.05)$ by Newman-Keuls test.

Tabela 7 - Teores médios de cálcio $(\mathrm{Ca})$, fósforo $(\mathrm{P})$, magnésio $(\mathrm{Mg})$, manganês $(\mathrm{Mn})$, sódio $(\mathrm{Na})$ e potássio $(\mathrm{K})$ das cinzas de fêmures de pintos de corte

Table 7 - Levels of calcium (Ca), phosphorus ( $P$ ), magnesium (Mg), manganese (Mn), sodium (Na) and potassium (K) in the femur ash of broiler chicks

\begin{tabular}{lcccrrr}
\hline L-Glu $(\%)$ & $\mathrm{Ca}^{*}(\%)$ & $\mathrm{P}^{*}(\%)$ & $\mathrm{Mg}^{*}(\%)$ & $\mathrm{Mn}^{*}(\%)$ & $\mathrm{Na}^{*}(\%)$ & $\mathrm{K}^{*}(\%)$ \\
$L-G l u(\%)$ & $\mathrm{Ca}^{*}(\%)$ & $P^{*}(\%)$ & $\mathrm{Mg}^{*}(\%)$ & $\mathrm{Mn}^{*}(\%)$ & $\mathrm{Na}^{*}(\%)$ & $K^{*}(\%)$ \\
\hline 5,0 & $30,63^{\mathrm{a}}$ & $20,71^{\mathrm{a}}$ & $0,87^{\mathrm{a}}$ & $0,0035^{\mathrm{a}}$ & $0,88^{\mathrm{a}}$ & $0,69^{\mathrm{a}}$ \\
7,5 & $29,03^{\mathrm{a}}$ & $21,91^{\mathrm{a}}$ & $0,85^{\mathrm{a}}$ & $0,0048^{\mathrm{a}}$ & $1,02^{\mathrm{a}}$ & $0,98^{\mathrm{a}}$ \\
10,0 & $32,90^{\mathrm{a}}$ & $21,86^{\mathrm{a}}$ & $0,89^{\mathrm{a}}$ & $0,0052^{\mathrm{a}}$ & $1,00^{\mathrm{a}}$ & $0,80^{\mathrm{a}}$ \\
12,5 & $32,53^{\mathrm{a}}$ & $15,36^{\mathrm{b}}$ & $0,62^{\mathrm{a}}$ & $0,0031^{\mathrm{a}}$ & $1,08^{\mathrm{a}}$ & $0,95^{\mathrm{a}}$ \\
\hline
\end{tabular}

* Médias, nas colunas, seguidas de letras minúsculas não diferem $(P>0,05)$ pelo teste de Newman-Keuls.

* Means, in each column, with the same small letters, do not differ ( $P>.05)$, by Newman-Keuls test.

retenção de cálcio das tíbias e dos fêmures, embora um dos principais componentes das PNC seja a osteocalcina, que possui alto poder quelante para cálcio (Hauschka \& Gallop, 1977).

As relações médias de $\mathrm{Ca}: \mathrm{P}$ das cinzas de tíbias e de fêmures encontram-se na Tabela 8 . Os dados das tíbias estão próximos de $2: 1$, que é a relação sugerida para um bom desenvolvimento ósseo. No

Tabela 8 - Relação cálcio e fósforo (Ca:P) das cinzas de tíbias e fêmures de pintos de corte, machos, aos 14 dias de idade

Table 8 - Ash calcium and phosphorus ratio (Ca:P) of tibia and femur of male broiler chicks at 14 days of age

\begin{tabular}{ccc}
\hline L-Glu(\%) & Tíbias Ca:p & Fêmures Ca:p \\
L-Glu (\%) & Tibia Ca:p & Femur Ca:p \\
\hline 5,0 & $2,07^{\mathrm{a}}$ & $1,48^{\mathrm{b}}$ \\
7,5 & $2,04^{\mathrm{a}}$ & $1,32^{\mathrm{b}}$ \\
10,0 & $2,18^{\mathrm{a}}$ & $1,50^{\mathrm{b}}$ \\
12,5 & $2,17^{\mathrm{a}}$ & $2,11^{\mathrm{a}}$ \\
\hline
\end{tabular}

* Médias, na coluna, seguidas de letras minúsculas iguais não diferem, pelo teste de Newman-Keuls.

* Means, in column, with the same small letter, do not differ ( $P>$.05) by NewmanKeuls test.

R. Bras. Zootec., v.31, n.1, p.411-416, 2002 (suplemento) entanto, somente com $12,5 \%$ de L-Glu é que a relação $\mathrm{Ca}: \mathrm{P}$ dos fêmures foi próxima de $2: 1$. Os demais níveis de L-Glu resultaram em relação $\mathrm{Ca}: \mathrm{P}$ inferior nos fêmures $(\mathrm{P}<0,05)$.

De acordo com Lofton et al. (1986), um desbalanceamento da relação $\mathrm{Ca}: \mathrm{P}$ pode acarretar prejuízo na absorção de um ou de outro elemento. Existem evidências de que dietas com alto teor de cálcio e fósforo e relação $\mathrm{Ca}: \mathrm{P}$ desproporcionais podem causar deficiência secundária de manganês, induzindo à perose e às anomalias de pernas.

\section{Conclusões}

Considerando o conjunto de parâmetros avaliados neste experimento, recomendam-se $12,5 \%$ de L-Glu como fonte de nitrogênio não-específico para atender as exigências dos pintos de corte aos 14 dias de idade e reduzir a incidência de problemas de pernas.

Os dados sugerem que a incidência de anomalias esteja diretamente relacionada com a fração orgânica do osso, uma vez que a fração inorgânica não foi afetada. 


\section{Literatura Citada}

ALBERS, G.A.A. Criação e resistência às doenças em avicultura. In: CONFERENCIA APINCO, 1994. Conferência... Campinas: 1994. p.3-14.

BRADFORD, M. A rapid and sensitive method for the quantification of microgram of protein utilizing the principle of protein dye binding. Analytical Biochemistry, v.72, n.1, p.248-254, 1976.

DESAI, R.K.; Van WIJNEN, A.J.; STEIN, J.L. et al. Control of 1,25 dihydroxyvitamin $\mathrm{D}_{3}$ receptor-mediated enhancement of osteocalcin gene transcription: effects of perturbing phosphorylation pathways by okadaic acid and staurosporine. Endocrinology, v.136, n.12, p.5685-5693, 1995.

ELKIN, R.G.; FEATHERSTON, W.R.; ROGLER, J.C. Investigations of leg abnormalities in chicks consuming high tannin sorghum grain diets. Poultry Science, v.57, n.3, p.757-762, 1978 .

EMMERSON D.A.; ANTHONY, N.B.; NESTOR, K.E. et al. Genetic association of selection for increased leg muscle and increased shank diameter with body composition and walking ability. Poultry Science, v.70, p.739-745, 1991.

FEATHERSTON, W.R.; ROGLER, J.C. Methionine-cystine interrelationship in chicks fed diets containing suboptimal levels of methionine. Journal of Nutrition, v.108, n.1, p.954-958, 1978.

GUIMARÃES, V.M.; MORAES, G.H.K.; FONSECA, J.B. et al. Efeitos do ácido L-glutâmico, L-prolina e L-lisina da dieta no desenvolvimento e incidência de problemas de pernas em pintos de corte. Revista da Sociedade Brasileira de Zootecnia, v.22, n.4, p.584-590, 1993a.

GUIMARÃES, V.M. Efeitos de aminoácidos dispensáveis e indispensáveis no desempenho e anomalias das pernas de pintos de corte. Viçosa, MG: Universidade Federal de Viçosa, 1988. 54p. Dissertação (Mestrado em Agroquímica) Universidade Federal de Viçosa, 1988.

GUIMARÃES, V.M.; MORAES, G.H.K.; ROSTAGNO, H.S. et al. Efeitos de aminoácidos não essenciais no desenvolvimento e incidência de problemas de pernas em pintos de corte. Revista da Sociedade Brasileira de Zootecnia, v.22, n.4, p.699-705, 1993b.

HAUSCHKA, P.V.; GALLOP, P.M. Purification and calciumbinding properties of osteocalcin, the gamma-carboxyglutamic acid-containing proteins of bone. In: WEISSIERMAN, R.H. (Ed.) Calcium binding proteins and calcium function. Amsterdan: Elsevier, 1977. p.338-347.

HONMA, N.H. Efeito dos níveis nutricionais de cálcio sobre a capacidade reprodutiva e integridade dos ossos de galos reprodutores de corte. Viçosa, MG: Universidade Federal de Viçosa, 1992. 63p. Dissertação (Mestrado em Zootecnia) - Universidade Federal de Viçosa, 1992.

LILBURN, M.S. Skeletal growth of commercial poultry species. Poultry Science, v.73, n.6, p.897-903, 1994.

LOFTON, J.T., SOARES JR., J.H. The effects of vitamin $\mathrm{D}_{3}$ on leg abnormalities in broilers. Poultry Science, v.65, n.5, p.749-756, 1986.

LONG, P.H.; LEE, S.R.; ROWLAND, G.N. et al. Experimental rickets in broilers: gross, microscopic, and radiographic lesions. I. Phosphorus deficiency and calcium excess. Avian Disease, v.28, n.2, p.460-475, 1983.
MORAES, G.H.K.; ROGLER, J.C.; FEATHERSTON, W.R. Effects of a nonspecific nitrogen deficiency on growth rate and leg problems in chicks. Poultry Science, v.63, n.2, p.344-353, 1984.

MORAES, G.H.K.; PERINI, D.T.; OLIVEIRA, T.T. et al. Efeitos do ácido L-glutâmico e vitamina $\mathrm{D}_{3}$ sobre o desempenho e anormalidades nas pernas de pintos de corte. Revista Brasileira de Zootecnia, v.26, n.4, p.773-778, 1997.

RIBEIRO, M.; MORAES, G.H.K.; FONSECA, J.B. Efeitos de fontes e níveis de nitrogênio não específico em dietas purificadas no desenvolvimento de pintos de corte. Revista da Sociedade Brasileira de Zootecnia, v.24, n.1, p.88-98, 1995a.

RIBEIRO, M.; MORAES, G.H.K.; FONSECA, J.B. Efeitos de ácido L-glutâmico, L-alanina e L-prolina da dieta em pintos de corte: I - Desempenho, incidência de problemas de pernas e composição química de fêmures. Revista da Sociedade Brasileira de Zootecnia, v.24, n.5, p.768-777, 1995b.

RODRIGUES, A.C.P.; MORAES, G.H.K. Efeitos do ácido L-glutâmico e da vitamina $\mathrm{K}$ da dieta no desempenho e nas anormalidades nas pernas de pintos de corte. Revista Ceres, v.42, n.248, p.270-278, 1995.

RODRIGUES, A.C.P.; MORAES, G.H.K.; ROSTAGNO, H.S. et al. Efeitos do ácido L-glutâmico e da vitamina $\mathrm{K}$ no comprimento e na composição química parcial de tíbias e fêmures de pintos de corte. Revista Ceres, v.43, n.249, p.567-580, 1996.

SASSE, C.E.; BAKER, D.H. Modification of the Illinois reference standard aminoacid mixture. Poultry Science, v.52, n.5, p.1970-1972, 1973.

SILVA, D.J. Análise de alimentos (métodos químicos e biológicos). Viçosa, MG: Universidade Federal de Viçosa, 1990. 166p.

TAFURI, M.L.; VILORIA, M.I.V.; FONSECA, J.B. et al. Observações preliminares sobre a composição de tecido ósseo e deformações de pernas de frangos de corte. Revista Ceres, v.40, n.231, p.491-501, 1993.

TARDIN, A.C. Visão nutricional dos problemas locomotores em frangos de corte. In: CONFERÊNCIA APINCO, 1995. Conferência... Campinas: 1995. p.71-83.

THIEDE, M.A.; SMOCK, S.L.; PETERSEN, D.N. et al. Presence of messenger ribonucleic acid encoding osteocalcin, a marker of bone turnover, in bone marrow megakaryocytes and peripheral blood platelets. Endocrinology, v.135, n.3, p.929937, 1994.

UNIVERSIDADE FEDERAL DE VIÇOSA - UFV. Sistema para análises estatísticas e genéticas. Versão 7.1. Viçosa, MG: 1997. 150p. (Manual do usuário).
Recebido em: 15/06/00 Aceito em: 10/10/01 